EPJ Web of Conferences 60, 16013 (2013)

DOI: $10.1051 /$ epjconf $/ 20136016013$

C Owned by the authors, published by EDP Sciences, 2013

\title{
Top quark pair properties: spin correlation, charge asymmetry and complex final states at LHC in ATLAS
}

\author{
Francesco Rubbo ${ }^{1, \mathrm{a}}$, on behalf of the ATLAS Collaboration \\ ${ }^{1}$ Institut de Física d'Altes Energies, Universitat Autònoma de Barcelona
}

\begin{abstract}
In proton-proton collisions at the LHC, pairs of top and anti-top quarks are expected to be mostly produced through gluon fusion, in contrast to production at the Tevatron, where quark annihilation dominates. Making use of the large number of top quark pairs, we present measurements of the spin correlation between top and anti-top quarks as well as of the top quark charge asymmetry, which constitute important tests of QCD and are sensitive to new physics. We also discuss top production in association of photons and $\mathrm{Z}$ bosons.
\end{abstract}

\section{Introduction}

At the LHC the top quark is produced mostly in pairs. Given its short lifetime $\left(\approx 5 \times 10^{-25} s\right)$, each top quark decays rapidly into a $W$ boson and a $b$ quark, before hadronization can occur. Hence top pair properties are directly accessible through its decay products. The decay modes of the two $W$ bosons define three possible signatures: the all hadronic channel, when both $W$ s decay into a quark and an anti-quark, is characterized by four lightquark jets and two b-jets; the dilepton channel, when both $W$ s decay into a charged lepton and a neutrino, is characterized by two opposite sign leptons, two b-jets and large missing transverse momentum; and the semileptonic channel with one hadronic and one leptonic $W$-boson decay, is characterized by one lepton, two jets, two b-jets and missing transverse momentum. The measurements described here are performed in dilepton and semileptonic channels using, partially or in full, the data set collected at the ATLAS experiment [1] during the $2011 \sqrt{s}=7 \mathrm{TeV}$ run.

\section{Top pair properties}

\subsection{Charge asymmetry}

The top charge asymmetry is defined as

$$
\mathrm{A}_{\mathrm{C}}=\frac{N(\Delta|y|>0)-N(\Delta|y|<0)}{N(\Delta|y|>0)+N(\Delta|y|<0)}
$$

where $\Delta|y|$ is the difference of the absolute values of the rapidity of top and anti-top. The SM predicts a small charge asymmetry $\mathrm{A}_{\mathrm{C}}=0.0115(6)[2]$ in $t \bar{t}$ production at LHC due to NLO interference effects in $q \bar{q} \rightarrow t \bar{t}$ events. The $\Delta|y|$ distribution is reconstructed (Fig. 1) and unfolded (Fig. 2) to the parton-level with an iterative bayesian procedure [3].

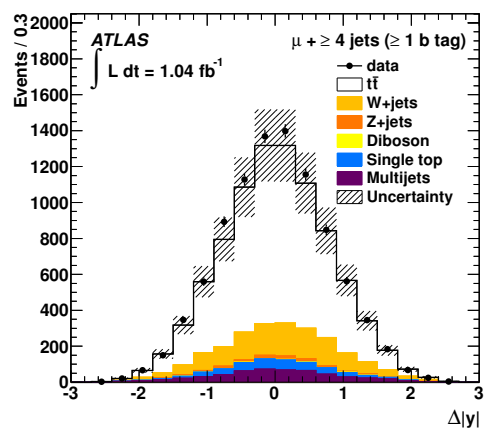

Figure 1. The measured $\Delta|y|$ distribution before unfolding for the muon channel after $b$-tagging is applied. Data (points) and predictions (solid lines) are represented. The uncertainty on the combined signal and background estimate includes both statistical and systematic contributions [4].

In the lepton+jets channel, the asymmetry is measured to be $\mathrm{A}_{\mathrm{C}}=-0.019 \pm 0.028$ (stat . \pm 0.024 (syst.), using a data set of $1.04 \mathrm{fb}^{-1}$ [4]. The asymmetry is also measured in two $m_{t \bar{t}}$ bins, below and above $450 \mathrm{GeV}$, as shown in Fig. 3.

Figure 4 shows how the ATLAS result is compatible with the SM prediction and disfavors a flavor changing Z' model.

In the dilepton channel, the top charge asymmetry is measured to be $\mathrm{A}_{\mathrm{C}}=0.057 \pm 0.024$ (stat.) \pm 0.015 (syst.), using a data set of $4.7 \mathrm{fb}^{-1}$ [7]. The combination of the two channels yields to $\mathrm{A}_{\mathrm{C}}=0.029 \pm 0.018$ (stat . \pm 0.014 (syst.).

In the dilepton channel, the lepton asymmetry is also measured. It is based on the lepton pseudorapidities and defined as

$$
A_{l}=\frac{N(\Delta|\eta|>0)-N(\Delta|\eta|<0)}{N(\Delta|\eta|>0)+N(\Delta|\eta|<0)}
$$




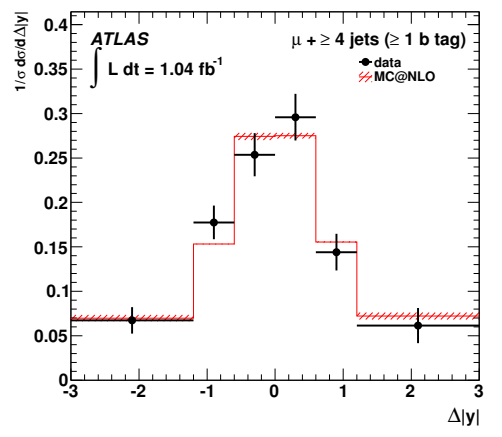

Figure 2. The unfolded $\Delta|y|$ distribution for the muon channel after b-tagging, compared to the prediction from Mc@NLO. The uncertainties on the measurement include both statistical and systematic contributions. The error bands on the Mc @ NLO prediction include uncertainties from parton distribution functions and renormalisation and factorisation scales [4].

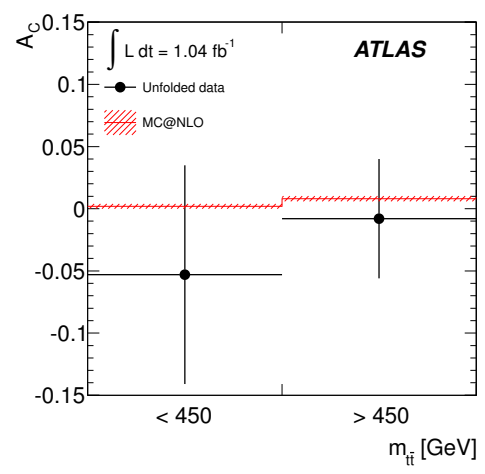

Figure 3. Unfolded asymmetries in two regions of $m_{t \bar{t}}$ compared to the prediction from Mc @ NLO. The error bands on the Mc@NLO prediction include uncertainties from parton distribution functions and renormalisation and factorisation scales [4].

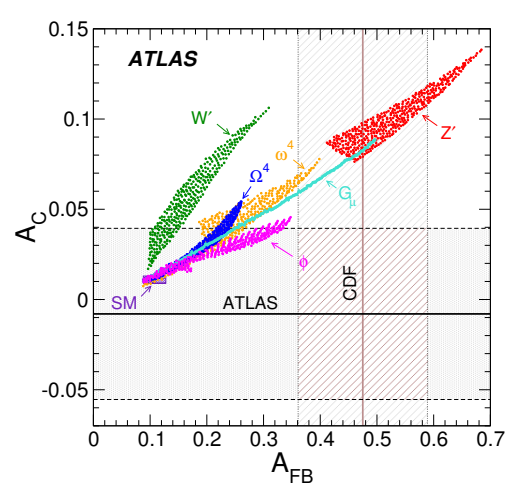

Figure 4. Measured FB asymmetry from the CDF and charge asymmetry from ATLAS [4], both for $m_{t \bar{t}}>450 \mathrm{GeV}$, compared to predictions from the $\mathrm{SM}$ as well as predictions incorporating various potential new physics contributions $[5,6]$.

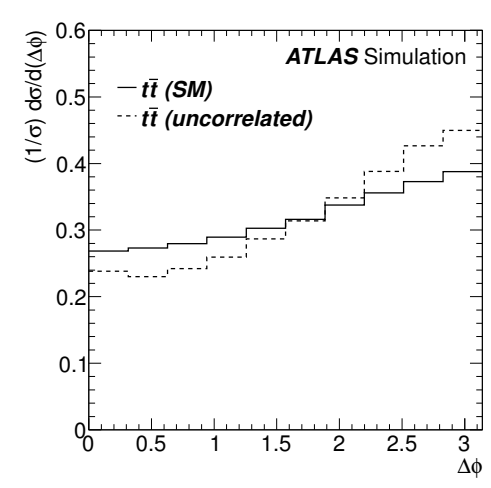

Figure 5. Normalized reconstructed charged lepton $\Delta \phi$ distribution for generated events at parton level for $\sqrt{s}=7 \mathrm{TeV}$ using Mc@NLo. The two histograms show the SM and uncorrelated spin scenarios [8].

The lepton asymmetry is measured to be $A_{l}=0.023 \pm$ 0.012 (stat.) \pm 0.008 (syst.).

No deviations from SM predictions are observed in either top charge asymmetry or lepton asymmetry.

\subsection{Spin correlation}

Since the top quark decays before hadronization, the decay products carry its spin information. Therefore the correlation between the top and the anti-top quark spin in top pair events can be measured. The degree of correlation is defined as

$$
A=\frac{N(\uparrow \uparrow)+N(\downarrow \downarrow)-N(\uparrow \downarrow)-N(\downarrow \uparrow)}{N(\uparrow \uparrow)+N(\downarrow \downarrow)+N(\uparrow \downarrow)+N(\downarrow \uparrow)},
$$

where $N(\uparrow \uparrow)$ and $N(\downarrow \downarrow)$ are the numbers of events with parallel spin states, while $N(\uparrow \downarrow)$ and $N(\downarrow \uparrow)$ represent antiparallel spin states. The size of $A$ in the SM depends on the relative fraction of $q \bar{q} / g g$ events and on the choice of the spin basis.

An observable which can be used to measure the spin correlation is the opening angle between the two leptons $(\Delta \phi)$ from the top pair dileptonic decay. The $\Delta \phi$ distribution in the correlated spin scenario (SM) is less steep than in the uncorrelated case, thus allowing a discrimination (Fig. 5).

Therefore the spin correlation is measured in the dilepton channel, using a data set of $2.1 \mathrm{fb}^{-1}$ [8]. The $\Delta \phi$ distribution is fit to a linear combination of uncorrelated and correlated predictions. Figure 6 shows the $\Delta \phi$ distribution measured in data compared with the correlated (SM) and uncorrelated $t \bar{t}$ predictions stacked on top of the background estimates.

The fitted fraction of correlated signal $f^{S M}$ is converted into the degree of spin correlation, $A$, in the helicity basis [9] and in the maximal basis [10] by multiplicative factors derived from MC. The helicity basis corresponds to the direction of flight of the top quark in the centre-of-mass frame of the $t \bar{t}$ system. The maximal basis is optimized for the $g g \rightarrow t \bar{t}$ contribution. 


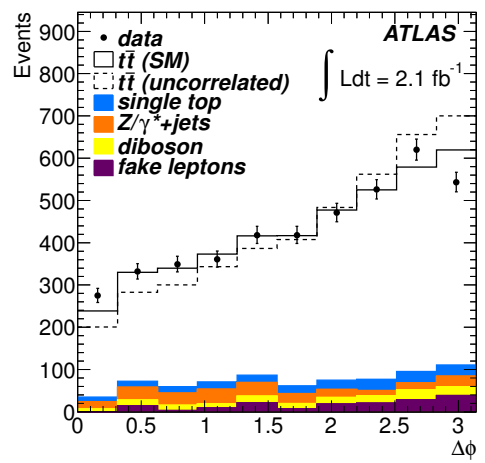

Figure 6. Reconstructed charged lepton $\Delta \phi$ distribution for the sum of the three dilepton channels. The integrated number of events for both the SM and the uncorrelated $t \bar{t}$ samples is fixed to the value from the fit [8].

The fitted value for $f^{S M}$ is $1.30 \pm 0.14$ (stat. $)_{-0.22}^{+0.27}$ (syst.), which translates into $A_{\text {hel }}=0.40 \pm 0.04(\text { stat } .)_{-0.07}^{+0.08}$ (syst. $)$ and $A_{\text {max }}=0.57 \pm 0.006(\text { stat } .)_{-0.10}^{+0.12}$ (syst. . .

The measured $f^{S M}$ is compatible with unity; hence the measured degrees of spin correlation are compatible with the SM predictions $A_{h e l}^{S M}=0.31$ and $A_{\max }^{S M}=0.44$. The measurement represents the first observation of spin correlation in $t \bar{t}$ events with a significance of $5.1 \sigma$.

\section{Associated production with $V$-bosons}

Studying top pair production in association with vector bosons allows a test of the electroweak coupling of the top quark in the SM. The measurement of anomalous couplings would be a strong hint of physics beyond the SM.

The measurement of $t \bar{t} \gamma$ inclusive cross-section and the search for $t \bar{t} Z$ production represent the first steps towards probing the coupling of the top quark with the photon and the $Z$-boson.

\subsection{The $t \bar{t} \gamma$ cross-section}

The $t \bar{t} \gamma$ inclusive cross-section is measured in the single lepton channel using a data set of $1.04 \mathrm{fb}^{-1}$ [11]. The measurement is performed as a template fit of the distribution of the photon isolation observable $p_{T}^{\text {cone20 }}$, which is the total transverse momentum of tracks in a cone of radius 0.2 around the reconstructed photon. Figure 7 shows how prompt $t \bar{t} \gamma$ events are characterized by isolated photons while the main background of fake photons from hadrons lies at high $p_{T}^{\text {cone20 }}$ values. Other smaller sources of background are fake photons from electron misreconstruction, non-prompt $t \bar{t} \gamma$ processes and non- $t \bar{t}$ background with real or fake associated photons. The templates for the main backgrounds with fake photons from hadrons and electrons are estimated with data-driven techniques.

The cross-section for $t \bar{t} \gamma$ process is measured to be $\sigma_{t \bar{\gamma} \gamma} \times B R=2.0 \pm 0.50($ stat. $) \pm 0.70($ syst $) \pm 0.08$ (lumi. $) p b$ for photons with transverse momentum greater than $8 \mathrm{GeV}$. The main sources of uncertainties come from the modelling of initial and final state radiation, jet energy scale

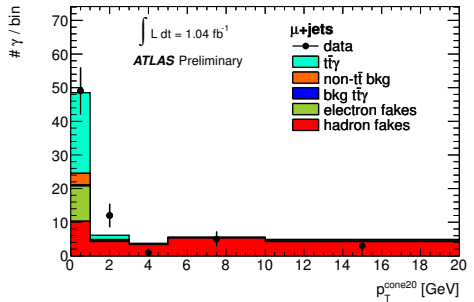

Figure 7. Result of the template fit using both channels, shown for the single muon channel. The predicted $t \bar{t} \gamma$ signal is shown on top of the different background contributions [11].

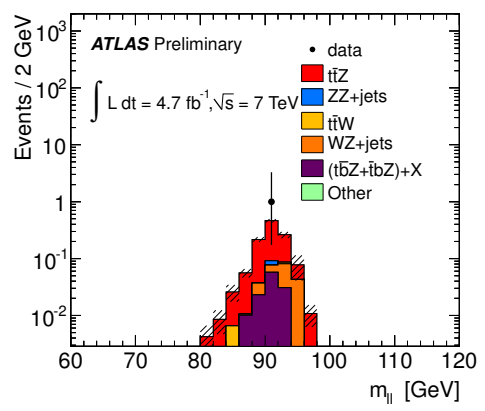

Figure 8. Expected and observed distributions of the OSSF pair of leptons [12].

and photon identification. The measurement of such crosssection corresponds to a $2.7 \sigma$ significance for $t \bar{t} \gamma$ process, and it is compatible with the SM prediction of $\sigma_{t \bar{t} \gamma} \times B R=$ $2.1 \pm 0.04 \mathrm{pb}$.

\subsection{Search for $t \bar{t} Z$ production}

The search for the production of a top pair produced in association with a $Z$ boson is performed in the three leptons + jets channel, where two of the leptons are required to be of opposite sign and same flavor (OSSF). The full 2011 data set of $4.7 \mathrm{fb}^{-1}$ is used [12].

The expected number of signal events is $0.85 \pm$ 0.04 (stat.) \pm 0.14 (syst.) with a background estimation of $0.28 \pm 0.05$ (stat. $) \pm 0.14$ (syst.). Figure 8 shows the distribution of the OSSF leptons invariant mass expected compared with the value measured in the single observed event candidate.

The observed event contains two muons of opposite charge and a single electron, compatible with a $Z$ boson decay and a $t \bar{t}$ semileptonic decay, respectively. The observation of a single candidate event corresponds to a limit on the $t \bar{t} Z$ cross-section $\sigma_{t \bar{Z} Z}<0.71 p b$ at a 95\% C.L., compatible with the expected SM cross-section of $0.14 \mathrm{pb}$.

\section{Conclusion}

The 2011 data set collected with the ATLAS detector was used to measure many top pair properties at $\sqrt{s}=7 \mathrm{TeV}$ proton-proton collisions. 
The charge asymmetry was measured inclusively as well as a function of $t \bar{t}$ invariant mass and found to be in agreement with SM predictions.

The first observation of spin correlation was achieved with a $5.1 \sigma$ significance and size compatible with the SM theoretical calculation.

The first measurement of $t \bar{t} \gamma$ cross-section was performed with a $2.7 \sigma$ significance as well as a fist search for $t \bar{t} Z$ production, setting a limit of about five times the SM expectation.

Most of the measurements are dominated by systematic uncertainties, even though the charge asymmetry differential measurement and the search for $t \bar{t} Z$ production will benefit from updates with the large 2012 data set. The effort of the ATLAS collaboration in constraining the source of uncertainties will allow more and more precise measurements of the top pair properties.

\section{References}

[1] ATLAS Collaboration, JINST 3, S08003 (2008)

[2] J. Kuhn, G. Rodrigo, Journal of High Energy Physics 1201, 63 (2012)
[3] G. D’Agostini, Nucl. Instrum. Meth. A 362, 487 (1995)

[4] ATLAS Collaboration, The European Physical Journal C 72 (2012)

[5] J.A. Aguilar-Saavedra, M. Pérez-Victoria, Phys. Rev. D 84, 115013 (2011)

[6] J. Aguilar-Saavedra, M. Pérez-Victoria, Journal of High Energy Physics 1109, 97 (2011)

[7] ATLAS Collaboration (2012), ATL-COM-PHYS-2012-057, http://cds. cern. ch/record/1453785

[8] ATLAS Collaboration, Phys. Rev. Lett. 108, 212001 (2012)

[9] G. Mahlon, S.J. Parke, Phys. Rev. D 81, 074024 (2010)

[10] P. Uwer, Physics Letters B 609, 271 (2005)

[11] ATLAS Collaboration (2011), ATL-COM-PHYS-2011-153, http: //cds. cern.ch/record/1398197

[12] ATLAS Collaboration (2012), ATL-COM-PHYS-2012-126, http://cds. cern.ch/record/1474643 